\title{
Patagium Rehabilitation Treatment in Wild Birds Following Long-term Wing Immobilization
}

\author{
S. Jato, PhD, PT, I. Otero, F. Gonzalez, DVM, I. Lopez, DVM, and J. L. Mendoza, DVM \\ GreFa WildLife Hospital \\ MADRID, SPAIN
}

\begin{abstract}
Patagium retraction is one of the most common problems after long-term periods of immobilization due to bandaging for injury treatment or loss of motion related to chronic pathology. Physiotherapy technics in protocolized treatments involve thermotherapy, laser, ultrasound, Z-massage, manual therapy, stretching, and kinesiotherapy. These treatments, followed by strength and endurance enhancement, allow improvement of full range of motion, propioceptive training, neuromuscular awareness, and avoid the development of fibrotic tissue, calcifications, and tie downs. The success of the evolution process was tested with goniometry, centimetric measurements, percentage score assessment, and imaging tools. This study was developed on a basis of 200 wild birds under treatment at the GREFA Wildlife Hospital of Madrid, Spain.
\end{abstract}

Key words: patagium, retraction, calcification, fibrosis, immobilization, physiotherapy technics, range of movement, rehabilitation program

\section{INTRODUCTION}

The patagia are double layers of skin in which the metapatagium runs from the medial surface of the upper wing to the lateral wall of the thorax and serves for insertion of the shoulder plumage. The propatagium stretches between the lateral surface of the upper wing and the medial surface of the lower wing. The patagia contain an elastic membrane that is stretched when the wing is extended and thus helps to return the wing to its resting position (Baumel et al 1979).

The propatagium, along with related feathers, is essential for producing aerodynamic flight. Propatagial tears or propatagial ligament injuries are very difficult to repair and can render the bird flightless. The propatagial tendon and the ligaments that run through this web of skin are important for flight (Lucas and Stettenheim 1972). These tendinous structures are

Susana Jato-Schez O'Shane is a physician specializing in emergency care and first aid. She has been GREFA's physiotherapist since 2005. She worked with Olympic athletes then began applying physical therapy protocols to horses, dogs, and cats. She later specialized in wildlife physiotherapy.

Ignacio Ptero has been GREFA Rehabilitation Manager since 2002. Now he participates in radio tracking black vultures in Madrid. Ignacio also coordinated the oiled bird rescue following the Prestige oil spill in 2002 composed of collagen or elastic connective tissue fibers and form their own intersecting web to reduce turbulence. The tendinous structures of the propatagium pose a problem for repair because elastic fibers do not hold sutures well and may result in undermining and translocation. Contraction occurs by immobilizing the wing for healing of propatagial tears or holes, and for fracture repair, which may result in an altered conformation of the leading edge of the wing or a wing that is incapable of full extension (Orosz 2002).

Tendons are framed within the general term tissue and their structure is characteristic of this genus: a small cell component that produces and maintains a more plentiful extracellular matrix. From the macroscopic viewpoint, healthy tendons are white and shiny, firm to the touch, but flexible (Józsa et al 1991). Microscopically, the main components are tendon cells (tenocites) and the extracellular matrix (collagen, elastin, and ground substance). In patagium retractions, the injury is due to the physiological vulnerability of poor perfusion (Kraushaar and Nirschl 1999).

The effects of immobilization on connective tissue have been widely reported in the literature. Use of early range of motion (ROM) exercises to prevent the effects of immobilization has become accepted practice. However, early exercise is not always possible and immobilization results in loss of tissue substrate with a subsequent loss of basic tissue components. The reversibility of these changes appears to be dependent on the length of the immobilization and the application of rehabilitation protocols.

When an injury occurs, two problems immediately arise that must be addressed. First is the generalized loss of physical fitness that occurs when activity is stopped, and second is the specific inactivity of the injured or adjacent parts, resulting from protective splinting of the soft tissue and, in some cases, immobilization by external means. 


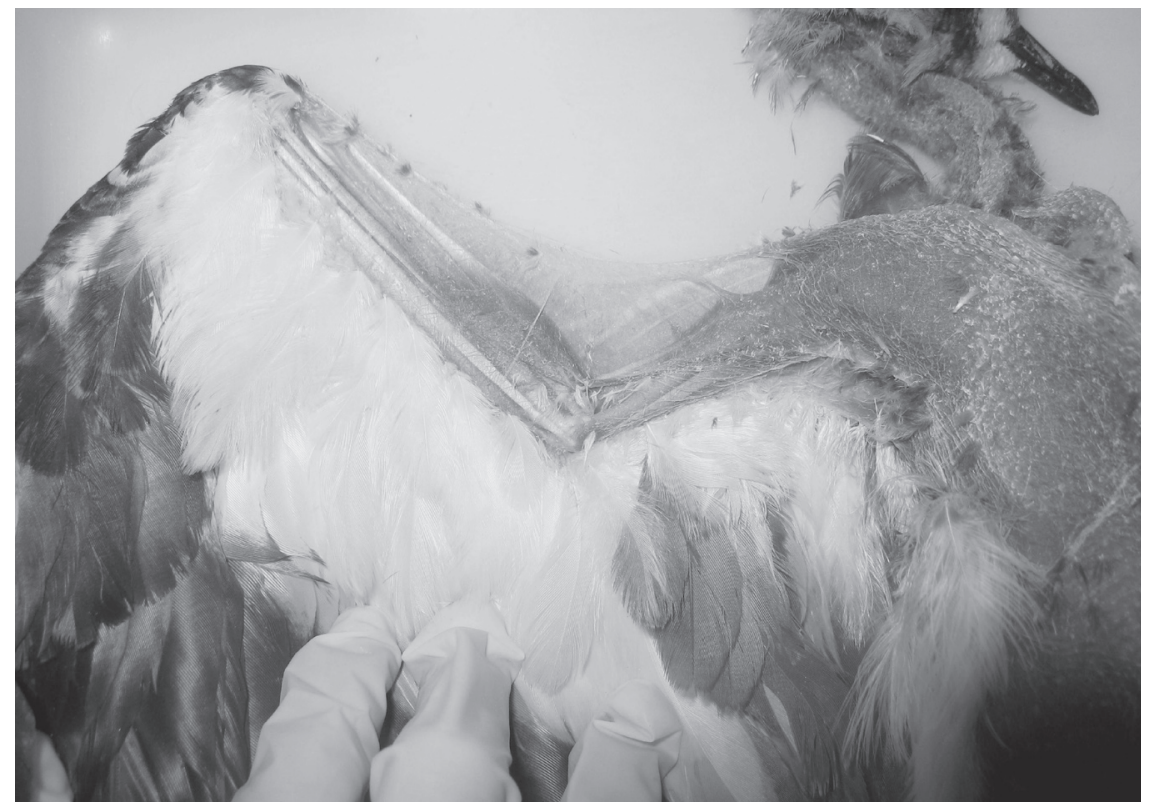

Figure 1. Patagium with normal extension.

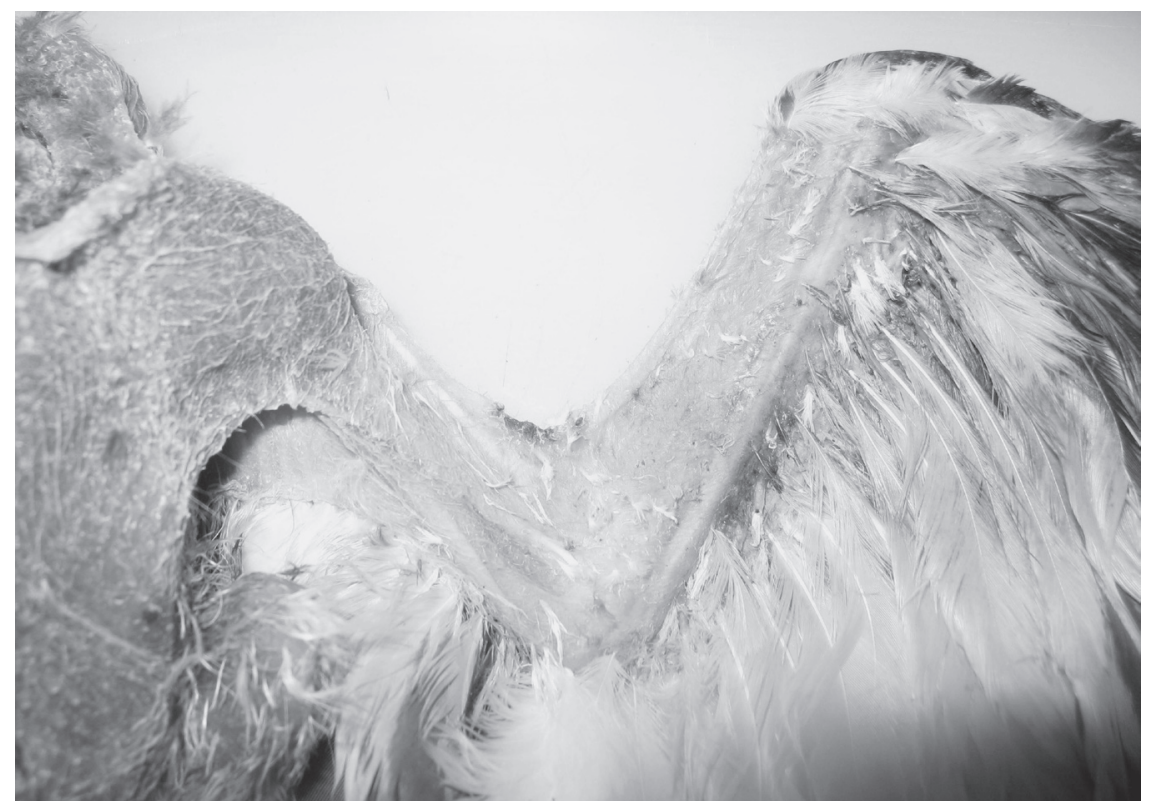

Figure 2. Patagium with retracted extension.

Immobilization for as short a period as 24 hours leads to definite adverse muscular changes (Dickinson and Bennett 1985). Disuse of the patagium results in atrophy, contracture, inflexibility, and delay in healing because of circulatory impairment. In addition to the physical aspect of the injury, the mental and emotional aspects of the bird also must be considered. Immobilizing the wing in a shortened position encourages atrophy and greater loss of contractile function. As the unused patagium decreases in size because of atrophy, protein also is lost. Immobilization decreases motor neuron discharge between 5 and 15 percent (Marino 1986). Additionally, when the pata- gium is immobilized, it loses its primary purpose, which is stabilization. Tendons respond to normal stress by maintaining their strength or becoming stronger; however, when stress is eliminated or decreased, they become weaker (Marino 1986).

\section{MATERIALS AND METHODS}

The application of heat to treat disease and traumatic injuries has been used for centuries. Heat is an energy form that increases molecular activity by four methods of transfer, including conduction, convection, conversion, and radiation (Michlovitz 1986). Thermotherapy modes are moist, dry, superficial, and deep.

For patagium treatment and as a first step, wet heat is used for 5 to 10 minutes over the affected area. Hot pack at $42^{\circ} \mathrm{C}\left(107.6^{\circ} \mathrm{F}\right)$ applied superficially to the patagium directly increases the subcutaneous temperature and indirectly spreads to the deeper tissues. Comparatively, when dry heat is applied at the same temperature, moist heat causes a greater indirect increase in the deep tissue temperature than does dry, so it is especially useful in plumage areas. Superficial heat application causes vasodilation that continues up to one hour after its removal. In general, application of a hot pack is a safe therapeutic medium for rehabilitation of the patagium when applied directly over the feathers. In a bird with lack of feathers, layers of towelling or commercial terrycloth must be used between the heat pack and the skin of the bird.

The desirable effects of heat include increasing the extensibility of collagen tissues, decreasing stiffness, reducing pain, relieving spasm, and increasing blood flow.

Heat affects the extensibility of collagen tissue by increasing the viscous flow of collagen fiber and subsequently relaxing the tension. Heating contracted connective tissue permits an increase in extensibility through stretching. Patagium fibrosis can be stretched effectively while being heated or just after the heat is removed (Greenberg 1972). An increase in extensibil- 
Table 1. GREFA Scale Degrees (centimeters) Quantitative Assessment of Patagium Retraction.

\begin{tabular}{|c|c|c|}
\hline Degree & Severity & $\%$ of Decrease* \\
\hline 0 & No Existence & $0 \%$ \\
\hline 1 & Very Mild & $0-10 \%$ \\
\hline 2 & Mild & $11-20 \%$ \\
\hline 3 & Moderate & $21-30 \%$ \\
\hline 4 & Severe & $31-40 \%$ \\
\hline 5 & Serious & $41-50 \%$ \\
\hline 6 & Very Serious & $>50 \%$ \\
\hline$\%$ of decrease $=($ A-B $) \times 100 / A$ \\
A = length in cm of patagium in healthy wing \\
B = length in cm of patagium in affected wing \\
$\begin{array}{l}\text { Centimeter patagium measurement = humeral head } \\
\text { (top edge) to major process carpal }\end{array}$
\end{tabular}

Table 2. Patagium retraction classification and description.

\begin{tabular}{|l|l|}
\hline Absent & $\begin{array}{l}\text { Good flexibility and elasticity } \\
\text { Good hydration and vascularization } \\
\text { Full ROM } \\
\text { No calcification } \\
\text { No fibrosis }\end{array}$ \\
\hline Very Mild & $\begin{array}{l}\text { Slightly dehydrated } \\
\text { Minimum resistance to extension } \\
\text { Pallor } \\
\text { ROM is complete with slight difficulty }\end{array}$ \\
\hline Mild & $\begin{array}{l}\text { Dehydration observable } \\
\text { Marked pallor } \\
\text { Strong resistance to extension } \\
\text { Moderate difficulty to complete the } \\
\text { ROM }\end{array}$ \\
\hline Moderate & $\begin{array}{l}\text { Dehydrated, compact } \\
\text { Poor vascularization } \\
\text { Resistance accused to the extension } \\
\text { Nodules Yes / No fibrotic } \\
\text { Unable to complete the ROM }\end{array}$ \\
\hline Severe & $\begin{array}{l}\text { Very dehydrated } \\
\text { Fibrosed } \\
\text { Signs of calcification } \\
\text { Lividity } \\
\text { Severe restriction of ROM } \\
\text { Plumage deteriorated }\end{array}$ \\
\hline Serious & $\begin{array}{l}\text { Calcification } \\
\text { Extreme fibrosis } \\
\text { Major restriction on movement } \\
\text { Hard limit } \\
\text { Loss of feathers }\end{array}$ \\
\hline $\begin{array}{l}\text { Extreme restriction of movement } \\
\text { Signs of ankylosis } \\
\text { Impaired neurovascular } \\
\text { Osteoarticular diminished or } \\
\text { abolished reflexes }\end{array}$ \\
\hline
\end{tabular}

ity does not occur unless heat treatment is associated with passive mobilization and stretching.

Heat also relieves pain, stimulating the free nerve endings and peripheral nerves by a "gating" mechanism (Rivenburg 1992), and is connected to a number of related factors, such as increasing metabolism, reducing oxygen tension, lowering $\mathrm{pH}$ level, increasing capillary permeability, and releasing histamine and bradykinin that cause vasodilation (Lehman and Delateur 1990).

After application of hot pack Global Mobilization (passive cinesitherapy) is realized to:

- Maintain and improve the mechanical properties of muscle and tendon structures

- Promote blood circulation and lymphatic, improvement of tissue nutrition

- Promote psychic stimulation when the patient is unable to move by itself

- Achieve propioceptive stimulation, maintenance of body schema and spacial

The therapeutic value of Z-Massage lies in its numerous and combined physiologic effects. Some effects are: increasing blood flow, reflex vasodilation, increasing diameter and permeability of capillaries, and manually separating fibers. These increase cellular nutrition, metabolism, toxin removal, and flexibility. It also decreases soreness, pain, fibrosis tendencies, spasms, undesired adhesions, and atrophy. Transverse scars interfere with normal contraction and strength and are subject to re-injury, chronic pain, and adherence to adjacent fibers. Adhesions between individual fibers limit contraction. The adhesions can cause chronic pain because of the variation of tension during contraction in the areas where normal tissue joins affected tissue.

To limit adhesions, Z-massage is used, as the arrangement of fibrils is dependent on mechanical factors, especially movement (Cyriax 1980). Thus appropriate movement within the tendon as it is healing inhibits unwanted adhesion formation and creates flexible tissue where needed. Z-Massage becomes a more important treatment modality since active movement is less effective in fiber spreading after immobilization periods.

Patagium retractions heal best with $\mathrm{Z}$-massage followed by stretching. For Z-massage to be effective the exact lesion site must be massaged and the manual forces of fingers must be at right angles to the fibers. To achieve this, the patagium must be in complete elongation and mild tension. The application begins over the proximal and distal extremes 
and progresses to the middle of the patagium, avoiding in the first sessions the centre of the tendinous structure to prevent leaking. Fingers must develop two perpendicular forces to the tendon and in opposite directions. Longitudinal friction or intempestive forces also must be avoided to prevent a roughening between the surface of the tendon and its sheath, and possible deposit of calcium.

Stretching or flexibility exercise utilizes forced but controlled movement to improve range of motion. Regardless of the type employed, motion should not be carried beyond the point of tightness (Knight 1985). Stretching involves holding a comfortable stretched position for various lengths of time. There should be no bouncing or other movement. The passive stretching of the wing (by manual assistance of the rehabilitator) is realized after the $\mathrm{Z}$-massage to reinforce the action on non-elastic fibers, as dense scar tissue interferes with normal blood flow and disturbs afferent (carrying toward) nerve input. The limit of stretching must be carefully observed by pulse rate, breathing rate, and reactions of the bird. Overstretching activates the stretch reflex, a monosynaptic reflex response that causes contraction of the muscle and tendon that are intended to be stretched. All overstretching is useless and injurious. Proper stretching elongates the muscle slowly to a point where a mild tension is felt. For an easy stretch, the elongated position is held 10 to 30 seconds. Stretching reduces muscle tension, improves flexibility, and reduces or prevents soreness. After an initial easy stretch, a fraction of an inch more stretch may be added for 10 to 30 seconds, but should not be painful for the bird or increase in intensity.

Following stretching, active exercise must be realized by the bird to maintain the improved motion. The basic progression of a therapeutic exercise program ranges from simple, less strenuous tasks to more complex and demanding tasks. The initial goal of an exercise program for the bird is to establish a full, pain-free range of motion through a detailed protocol of heat, cinesitherapy, Z-massage, stretching, ultrasound, and/or laser therapy. As the range of motion nears that of the opposite wing (i.e., normal), exercises designed to improve strength are initiated. When strength has been achieved, exercise for developing power and endurance are begun. The final stage of rehabilitation is incorporation of activity to improve coordination and provide re-education of muscles in the injured area. Each goal is dependent upon successful completion of the previous one.

Low level laser also is referred to as a biostimulation laser, soft laser, or healing laser. Therapy with laser is a form of intensive light therapy, which uses directed light of a defined wavelength and various frequencies to produce positive physiological cell changes and support the organism in its healing process. In patagium treatment, laser is used for wound healing, tissue regeneration, and stimulation. The energy used for patagium retraction is emitted for an Infrared diode laser unit of $700 \mathrm{~mW}$ and length wave of 785 $\mathrm{nm}$. For 3 minutes and 20 seconds, the injured patagium receives $10 \mathrm{~J} / \mathrm{cm}^{2}$ in CW (continuous wave) with the 'shower' applicator. This dosage activates biochemical, functional, and neurological processes such as promoting microcirculation by increasing vasodilation, activating immune cells by increasing leukocyte mobilisation, promoting lymphatic flow, and increasing production of adenosine triphosphate (ATP) and thus energy resources of the cell (Lundeberg and Malm 1991). In a damaged patagium, the regeneration of the tissue generated by laser application and the increased rate of mitosis and collagen synthesis, plus activation of fibroblasts (Basford 1995), combined with the production of ATP, and reduction of scars (Mester et al 1985), avoids fibrosis and calcification. Additionally, the laser treatment increases microcirculation and increases hyaluronidase activity.

The treatment periods depend on the effective performance of each laser system, so manufacturer's specification must be understood. Today, there are mainly continuously emitting lasers (CW) and pulsed lasers on the market. Continuous wave laser diodes entered the market in 1990s and are used in nearly every low energy laser therapy system today. Patagium therapy must orient to physiological laws and cannot simply take over technical logic (namely, higher intensity $=$ lower treatment times). The cell requires a minimal reaction time to absorb and integrate stimulation. Stimulous outside this 'therapeutic window' inhibits, destroys, or has no effect at all.

In the treatment of birds, a dense feather coat causes high intensity losses due to reflection (as well as dirt). This means that birds' therapy for patagium is always linked to longer treatment times. In order to treat larger areas, like the patagium, a laser shower applicator is used. The laser must strike perpendicular to the patagium surface. An inclined entry angle can increase reflection and refraction by approximately 40 to 50 percent and simultaneously reduce transmission. It is necessary to remove intermediate feather layers prior to treatment and feathers and skin must not be wet or dirty.

Therapeutic ultrasound is a deep heating modality that produces a sound wave of $1 \mathrm{MHz}$. The sound wave is produced by applying a high frequency, alternating electrical current to a natural quartz or syn- 
thetic crystal. This converts the electrical current into a mechanical vibration, a reversal of the piezoelectric effect (Licht 1965). Because of the increased extensibility ultrasound produces in tissues of high collagen content, it is an ideal modality for increasing mobility in those patagia with restricted range of motion (Enwemeka 1989). Sonation of a patagium must be combined with passive stretching to gain permanent motion in tight tissues, with stretch applied after the treatment has ended (Draper and Ricard 1995).

In addition to increasing collagen tissue extensibility, ultrasound also increases blood flow to the area as the bird attempts to cool overheated tissue. This action is useful in the resolution of fibrosis in tendon sheaths (Jackson et al 1991). Heating of tissues is responsible for the temporary increase in nerve conduction velocity. The stirring and streaming of molecules in the path of the sound wave is a non-thermal effect responsible for the positive effect of ultrasound on chronic wounds and is the basis for the phonophoresis (Cameron and Monroe 1992).

Ultrasound therapy longitudinal application mode of $1 \mathrm{MHz}, 0.1 \mathrm{~W} / \mathrm{cm}^{2}, 1: 10$ pulsing for 5 minutes is the modality of choice when fibrosis of the patagium appears. Because ultrasound causes an increase in local blood flow and enzymatic activity, it should not be applied in high doses as high doses cause calcium deposits. The vibrational qualities of ultrasound dictate that it should not be applied to the epiphyseal plates of birds or to unhealed surgical sites where the movement of adjacent molecules would retard or damage on-going healing processes.

In order for the sound wave to be propagated forward it must pass through a coupling agent to make a connection between the applicator and the patagium. Commercially prepared gel is the most efficient in transmitting sound waves, but as gel may be unpleasant on feathers, transmission is accomplished by a water-filled glove. A latex glove is filled with water until air is removed, tied shut, and placed under the sound head and over the patagium with a thin layer of gel between the water glove and the sound head.

The rehabilitator moves the sound head in circular, overlapping strokes over the patagium, from distal to proximal in a longitudinal way. Stationary application of ultrasound is discouraged because of the rapid temperature rise creating hot spots and increasing the possibility of blood clot formation (Oakley 1978).

Study Participants. All patients included in this study were evaluated through:

- Radiographs

- Centimetric measurement
- Goniometry

- Thermography (Thermographic camera model FLIR ThermaCam E45 ${ }^{\mathrm{TM}}$, FLIR Systems Inc., Boston, US)

- Outdoors trials (flight training)

Selection of patient participants implied quantitative and qualitative loss of functionality of patagium due to wounds, long-term bandages, luxations, fractures, or external surgical fixations. Birds with ankylosis belonging to breeding or educational programs also were included.

The importance of muscular fitness for successful performance in wild birds has led to widespread training and physical conditioning programs to supplement the skill training. These outdoors trials allow evaluation of patagium injuries as these injuries cause loss of strength, endurance, and power. Understanding how to assess various components of fitness can be useful in the following ways.

1. To evaluate the bird's potential for a particular environment

2. To plan a supplementary program of physiotherapy and physical conditioning to improve muscular performance

3. To pinpoint imbalances due to retractions, calcification, or fibrosis that lead to an increased risk of injuries after release

4. To determine loss of function due to injury

Treatment Protocol. Treatment protocol applied was scheduled three days per week for maximum of 30 minutes per day (to avoid stress) in a silent, calm area with a warm atmosphere. All birds wore falconry hoods or equivalent devices. Use of anaesthesia was avoided unless painful retractions had occurred or if the particular species stressed easily.

1. Hot pack to $42^{\circ} \mathrm{C}\left(107.6^{\circ} \mathrm{F}\right)$ over selected area for 5 to 10 minutes

2. Passive Cinesitheraphy: global mobilization of upper limb (flexion-extension to the firm limit) for 5 minutes

3. Manual treatment: Z-massage (perpendicular and opposite forces) for 2 to 3 minutes

4. Stretching (series of consecutive steps to the hard limit) for 5 to 10 minutes

5. Lasertherapy Las-Expert Physiomed ${ }^{\mathrm{TM}}$ (Physiomed Elektromedizin AG, Schnaittach/Laiperdorf, Germany) unit infrared diode with wavelength of 785 and power energy of $700 \mathrm{~mW}$ (Continuous wave-CW in 'shower' application $785 \mathrm{~nm} \mathrm{10J/}$ $\mathrm{cm}^{2} 3 \mathrm{~min} 20 \mathrm{sc}$ ) 
6. If fibrosed, ultrasound

(Physioson-Expert Physiomed $^{\mathrm{TM}}$

(Physiomed Elektromedizin

AG, Schnaittach/Laiperdorf,

Germany) therapy longitudinal

application $1 \mathrm{MHz} 0.1 \mathrm{~W} / \mathrm{cm}^{2}$

1:10 pulses for 5 minutes

7. Active cinesitherapy (after treatment in flight cage)

The centimetric valoration of the patagium is the measurement of the length of patagium from shoulder to carpal bones (Figure 3). The anatomical references are the glenoid fossa and first digit, phalanges 1 and 2 (alula basis or 'thumb' finger). The quantitative measurement from glenoid fossa to alula is compared with opposite normal patagium measurement in centimetres. This numeric value allows establishment of several categories of retraction. Plus, the sense and sensibility of palpation allow detection of typical signs of retraction and different grades of severity.

Goniometric measurement was used to determine both the particular joint position of the bird's elbow and the total amount of motion available at that joint, linked directly to the extensibility of patagium (Figure 4). Testing the passive ROM provides the examiner with information about integrity of the articular surfaces and the extensibility of the joint capsule, associated ligaments and tendons, and muscles.

The extent of the passive ROM is determined by the unique structure of the joint being tested. In patagium retractions, the tendon, fibrosis, and muscle tension limit the end of ROM. These have a characteristic feel that may be detected by the examiner performing the passive ROM. This feeling, which is experienced as a resistance to further motion at the end of a passive $\mathrm{ROM}$, is called the end feel. The examiner's ability to distinguish among different types of end feels requires practice and sensivity. Thus, determination of the end feel in retraction of patagium always was performed by the same GREFA physiotherapist. Cyriax (1977) has described a variety of physiologic and pathologic end

feels (soft, firm, hard, and empty). In retractions of the patagium and fibrosis, the end feel is soft; in calcification of patagium, it is firm. The quantitative measurement in degrees is obtained by bilateral comparison in the standard way:

- Body of the goniometer over the elbow

- Stationary arm perpendicular to the elbow

- Moving arm over axis of the ulna

Thermography records surface temperature and can measure large territories of skin temperature simultaneously. The method used is electronic. 


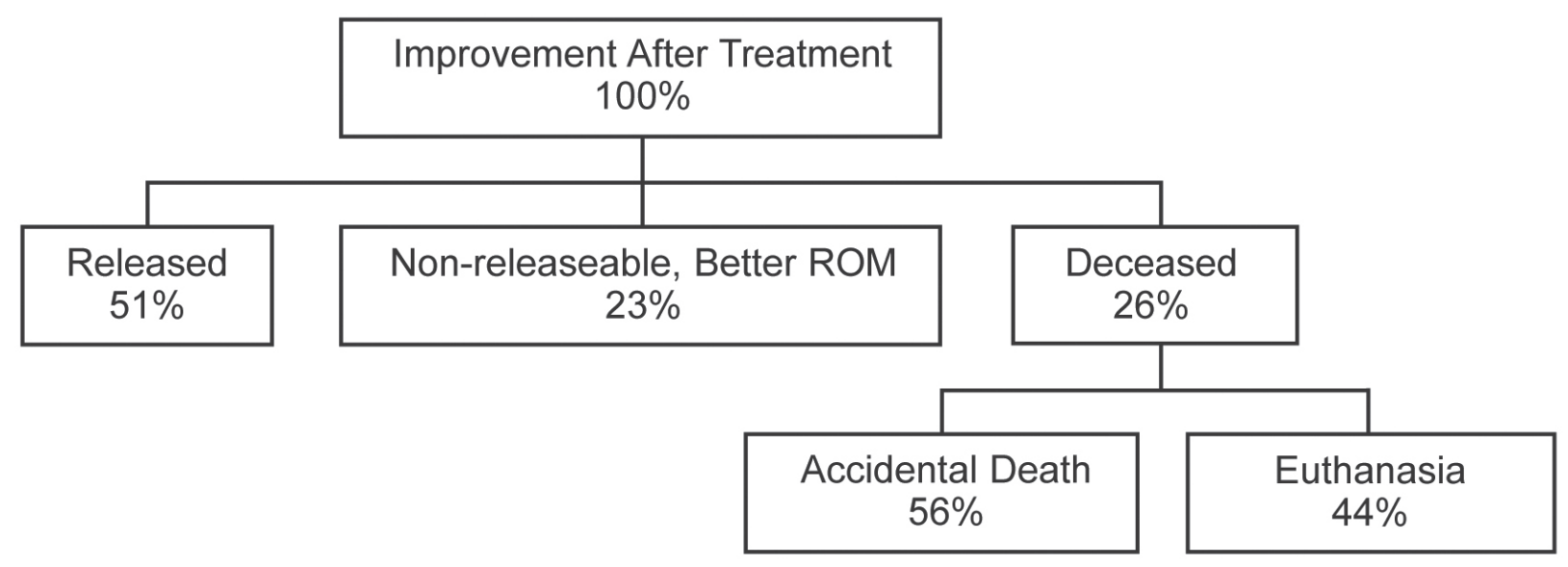

Recording the surface temperature of the patagium is very important to evaluate lesion and progression of rehabilitation program. The patagium surface temperature is primarily the result of the state of relaxation of the dermal microcirculation. The importance of thermography in training lies in the muscles, joints, ligaments, tendons, and bones, which have an embryonic somatocutaneous referral area. When nociception occurs in a somatic organ, the perception of pain is projected cutaneously to specific projection areas (Kellgreen 1937-1938; Anderson et al 2002; Green et al 1986). Regional hypothermia is the most expected and most important finding in study of patagiums affected by long term immobilization. This vasoconstrictor response to injury has been termed reflex sympathetic dysfunction and is associated with intense pain. Birds displaying such responses take longer to return to full performance. Measuring the territorial area of injury for the absence of heat and determination of severe vasoconstriction, with loss of normal surface heat, therefore is important. Thermographic examination allows estimating the severity of the injury, monitoring its evolution, and estimation of the healing time and eventual resumption of activity.

\section{CONCLUSION}

Rehabilitation or reconditioning is a dynamic method or program of prescribed exercise to prevent or reverse the deleterious effects of inactivity in birds before returning to the natural environment. The program also includes protocols and evaluation of return to a performance level equal to or higher than that of the pre-injury state. The goals of rehabilitation of the patagium in conjunction with the use of therapeutic modalities include decreasing pain, return of full, pain free, active range of motion, return of muscular strength, power, and endurance, and asymptomatic return to fully functional activities.

Early exercise is essential in restoring birds to a conditioned level as soon as possible, but a fine balance of patagium exercise must be achieved between early mobilization and that which is too aggressive, which can set back the rehabilitation process. In designing a rehabilitation program for the patagium, the rehabilitator must consider the arthrokinematics and tissues that have been traumatized or surgically repaired. In addition, harmful stimuli (an early flight trial, an intempestive stretching before proper healing of patagium) can result in pain, swelling, and inflammation. The initial phases of patagium rehabilitation should be concerned mainly with reducing these responses to injury.

All birds included in this study improved ROM, flexibility, and functional movement of patagium (Table 3), tested by methods described before and with specific work of rehabilitation in the field (flight). Birds with optimal response in trials were released. Some of the birds were tracked by GPS (Spanish imperial eagle, Aquila adalberti) to confirm the successful application of protocol and well-being after release. Non-releaseable birds due to other injuries were destined for educational programs, reproduction programs, or have become adult role models for future incoming birds in GREFA Hospital.

\section{LITERATURE CITED}

Anderson, J. L., S. I. Head, C. Rae, and J. W. Morley. 2002. Brain Function in Duchenne Muscular Dystrophy. Brain, A Journal of Neurology. 125(1): 4-13. 
Baumel, J. J., A. S. King, A. M. Lucas, J. E. Breazille, and H. E Evans (editors). 1979. Nomina Anatomica Avium. Academic Press: New York, NY.

Basford, B. R., 1995. Low Intensity Laser: Still Not an Established Clinical Tool. Lasers in Surgery and Medicine. 16: 331-342.

Cameron, M. H., and L. G. Monroe. 1992.

Relative Transmission of Ultrasound by Media Customarily Used for Phonophoresis. Physical Therapy. 72: 142-148.

Cyriax, J. 1977. Textbook of Orthopaedic Medicine. Treatment by Manipulation, Massage and Injection. Baliére Tindall: London, Gt. Britain.

Cyriax, J. 1980. Clinical Applications of Massage. Pp. 55-75 in Manipulations, Tractions and Massage, 2nd edition (J. B. Rogoff, editor). Williams \& Wilkins: Baltimore, MD.

Dickinson, A., and K. M. Bennett. 1985. Therapeutic Exercise. Pp. 25-48 in Symposium on Rehabilitation of the Injured Athletes, Clinics and Sports Medicine, Volume 14, No. 3 (J. S. Harvey, editor). W. B. Saunders Co: Philadelphia, PA.

Draper, D. O., and M. D. Ricard. 1995. Muscle Following $3 \mathrm{MHz}$ Ultrasound: The Stretching Window Revealed. Journal of Athletic Training. 30: 304-307.

Enwemeka, C. S. 1989. The Effects of Therapeutic Ultrasound on Tendon Healing: A Biomechanical Study. American Journal of Physical Medicine and Rehabilitation. 68: 283-287.

Green, J., A. Reilly, et al. 1986. Sympathetic Skin Response Abnormalities Correlated with Abnormal Infrared Thermogram. Pp 89-92 in Academy of Neuromuscular Thermography, Clinical Proceedings. Modern Medicine. New Jersey.

Greenberg, R. S. 1972. The Effects of Hot Packs and Exercise on Local Blood Flow. Physical Therapy. 52: 273-278.

Jackson, B. A., J. A. Schwanne, and B. C. Starcher. 1991. Effects of Ultrasound Therapy on the Repair of Achilles Tendon Injuries in Rats. Medicine and Science in Sports and Exercise. 23: 171-176.

Józsa, L., P. Kannus, and J. B. Bálint, et al. 1991. Three Dimensional Ultrastructure of Tendons. Acta Anatomica. 142: 306-312.

Knight, K. J. 1985. Guidelines for Rehabilitation of Sport injuries. Rehabilitation of the Injured Athlete. Clinics in Sports Medicine, Volume 4, No. 3 (J. S. Harvey, editor). W. B. Saunders Co: Philadelphia, PA.
Kellgreen, J. H. 1937-1938. Observation on Referred Pain Arising from Muscle. Clinical Scence. 3: 176.

Kraushaar, B., and R. Nirschl. 1999. Tendinosis of the Elbow. Clinical Features and Findings of Histological, Immunohistochemical, and Electron Microscopy Studies. Journal of Bone and Joint Surgery, America. 81(2): 259-278.

Lehman, J. D., and B. J. Delateur. 1990. Therapeutic Heat. Therapeutic Heat and Cold (J. F. Lehman, editor). Williams and Wilkins: Baltimore, MD.

Licht, S. 1965. Therapeutic Heat and Cold, 2nd edition. Waverly Press: Baltimore, MD.

Lucas, A. M., and P. R Stettenheim. 1972. Avian Anatomy: Integument. Agriculture Handbook. 362: 1-340. US Government Printing Office: Washington, DC.

Lundeberg, T., and M. Malm. 1991. Annals of Plastic Low Power He Ne Laser Treatment of Venous Leg Ulcers. Surgery. 27: 537-539.

Marino, M. 1986. Current Concepts on Rehabilitation in Sport Medicine: Research and Clinical Interrelationships. The Lower Extremity and Spine in Sports Medicine, Volume 1 (J. A. Nicolas and E. B. Hershman, editors). C. V. Mosby Co: St. Louis, MO.

Mester, E., A. F. Mester, and A. Mester. 1985. The Biomedical Effects of Laser Application. Lasers in Surgery and Medicine. 5: 31-39.

Michlovitz, S. L. 1986. Biophysical Principles of Heating and Superficial Heat Agents. Thermal Agents in Rehabilitation (S. L. Michlovitz, editor). F. A. Davis Co: Philadelphia, PA.

Oakley, E. M. 1978. Dangers and Contraindications of Therapeutic Ultrasound. Physiotherapy. 64: 173-174.

Orosz, E. 2002. Avian Surgical Anatomy: Thoracic and Pelvic Limbs. Saunders Company: Philadelphia, PA.

Rivenburg, D. W. 1992. Physical Modalities in the Treatment of Tendon Injuries. Clinics in Sports Medicine. 11: 645-659.

\section{RECOMMENDED READING}

Bockstahler, B., D. Levine, and D. Millis. 2004. Essential Facts of Physiotherapy in Dogs and Cats: Rehabilitation and Pain Management. BE VetVerla: Babenhausen, Germany.

Kottke, F., and J. Lehman. 1993. Krusen Medicina Física y Rehabilitación. Editorial. Médica Panamericana. 
Levine, D., D. Millis, D. J. Marcellin-Little, and R. Taylor. 2005. Veterinary Clinics of North America: Small Animal Practice. Rehabilitation and Physical Therapy. Saunders, Elsevier, Inc., Division: Philadelphia, PA.

McGowan, C., L. Goff, and S. Narelle. 2007

Animal Physiotherapy: Assessment, Treatment and

Rehabilitation of Animals. Blackwell Publishing: Victoria, Australia.

Proctor, N., and P. Lynch 1993. Manual of

Ornithology. Edwards Brothers, Inc: Ann Arbor, MI.

\section{ACKNOWLEDGEMENT}

Thank you to Mar Melero Asensio, veterinary student, and José M. Sánchez-Vizcaíno, $\mathrm{PhD}$, of the Animal Health Department, University Complutense of Madrid, for their collaboration in thermography items, and to Isaac Aizenman, IGA Medical Equipment and delegate of Physiomed Elektromedizin in Spain, for his technical support

of this study. (N.

\section{FROZEN: RATS, MICE \& CHICKS}

shipped anywhere in the USA www.frozenrodents.com

PERFECT PETS INC. 23180 SHERWOOD

BELLEVILLE, MI 48111

(800) 366-8794 